\title{
Exploring the User Experience of Proxemic Hand and Pen Input Above and Aside a Drawing Screen
}

\author{
Ilhan Aslan, Björn Bittner, Florian Müller, and Elisabeth André \\ Human-Centered Multimedia Lab, Augsburg University \\ Augsburg, Germany \\ lastname@hcm-lab.de
}

\begin{abstract}
Digital drawing experiences are not only fused by the flexibility of digital materials but also influenced by the availability of interaction space. In this paper, we first present a prototype, which implements a method to turn the (mid-air) space above and aside a drawing screen in a desktop setting dynamically into sensory space for gestural and spatial input. Then we report on a user study exploring how participants experience digital drawing when the additional interaction space above and aside a screen is exploited for exemplary proxemic input techniques for zooming and panning a drawing. Our results show that the new multimodal input techniques are perceived as significantly more attractive than a baseline drawing condition which only utilizes touch based input. We conclude by discussing implications and limitations of our findings and input above and aside a drawing screen in general.
\end{abstract}

\section{CCS Concepts}

-Human-centered computing $\rightarrow$ Interaction techniques;

\section{Author Keywords}

Interaction Technique; Bi-manual; Pen and Mid-air Gestures; Proxemic Input; User Experience

\section{INTRODUCTION}

Technology has influenced the practice of drawing by utilizing and transforming existing mental models of drawing materials, such as canvases, pens, and colors. Digital counterparts of such drawing materials are more flexible and enable the implementation of new crafting techniques and modalities, such as easily zooming into a (digital) canvas.

How we draw on screens is inspired by how we draw on paper and users' mental models are often constrained by the materiality of the original physical drawing materials and the original form of contact-based pen and paper interaction. For example, performing pen or hand movements above and around a drawing canvas without touching the canvas may have been

(C) $2018 \mathrm{ACM}$. This is the author's version of the work. It is posted here for our personal use. Not for redistribution. The definitive Version of Record can be found at:

DOI: https://doi.org/10.1145/3282894. 3282906 meaningless in the past, but are not necessarily meaningless anymore and may even become a widely recognized way of interacting with digital canvases in a foreseeable future.

Over the course of the last decade, touch-less and spatial interaction has indeed become common and is establishing as one of many ways to address the interface bottleneck (e.g., limited interface space on small sized screens) by contextually expanding the sensory space around a user or a device for spatial mid-air interaction (e.g., [9, 22, 28, 12]).
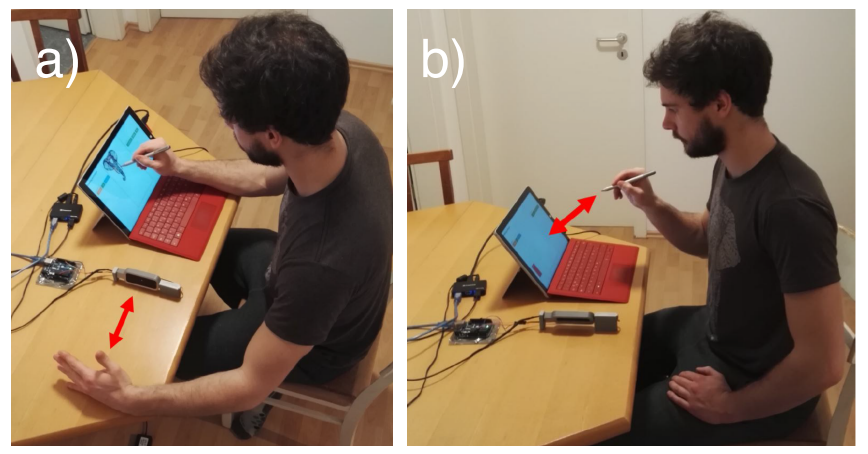

Figure 1. Overview of input techniques: a) hand-based proxemic interaction aside the screen/canvas and $b$ ) pen-based proxemic interaction above the screen/canvas

In this paper, we describe a prototype consisting of a touch device (i.e., Microsoft Surface Laptop), a Leap Motion 3D midair input controller, and an Arduino board controlling a servo motor to contextually actuate the Leap Motion's orientation either aside the touch screen device or above (see Figure 1). The aim of the prototype was to have a (multimodal) system which would allow the exploration of user experiences of drawing related input with a pen on a touch screen (i.e., a digital canvas), which is capable to sense proxemic input aside and above the screen prior to or in combination with touch.

The research question we address is how users experience interactions aside and above the canvas in combination with touch (or as an alternative to touch) in a drawing related task. That is, our aim is to explore the UX of a potential future alternative interaction technique for drawing.

In the next section, we provide background in multimodal and mid-air input. Then we present exemplary interaction techniques, which we implemented for input above and aside the 
Surface's screen, which is enabled by the multimodal prototype. Afterwards, we report on a user study with 20 participants comparing the UX of the novel multimodal solution with the traditional (unimodal) touch-based input as a baseline. Participants rated the multimodal alternative significantly higher than the traditional unimodal alternative considering hedonic qualities (e.g., overall attractiveness of the technique) while no differences were found considering pragmatic qualities (e.g., perceived usability).

\section{BACKGROUND}

Human-computer interfaces based on optical tracking systems have been gaining popularity, since they can transform "any" mid-air space into sensory space for human-computer interaction. The idea behind optical tracking systems is that cameras capture images of the space in front of them and are capable to recognize a user's body (or a specific body part such as a hand) located in these images. Then, information about the body, such as posture and the shape the body created during movement within the "digitalized" space is computed. Ultimately, the technology allows body-based mid-air interaction with computers, such as swipe and zoom hand gestures performed by users in mid-air.

Previous work has extensively explored mid-air input as a modality for human-computer interaction and designed its use for contexts, such as cars [10], clean rooms in industrial settings [14], and for retail situations [11]. Moreover, fellow researchers have highlighted benefits of mid-air input when used to complement other input modalities, such as finger based touch input or pen-based input in bi-manual interaction setups (e.g., $[8,7,26,25])$.

However, there are some common and systematic constraints of off-the shelf controllers, such as the Xbox Kinect or the Leap Motion. As optical systems they have specific requirements on orientation and proximity towards the in-build sensors. Thus, they require users to perform gestures in a limited and invisible sensory space, causing discomfort and unintended interactions. The colloquial term "the gorilla arm syndrome" refers to associated issues with fatigue and discomfort when postures have to be maintained over long periods and performed in a repetitive manner (e.g., [20, 34]).

Related research (e.g., $[18,21])$ has argued that allowing users to rest their arms (e.g., on a chair armchair) while performing hand gestures would limit the inherent effects of gestural midair interfaces. Motivated by these results in related research Aslan et al. [9] have propose a method to dynamically extend the sensory space of a 3D controller. They have argued that doing this not only would enable users to take rest positions, but also creates new use contexts (i.e., mid-air interaction aside or above the screen) and allows "seamless" transitions between them.

\section{Multimodal Input}

The benefits of using multi-touch and pen input in tandem on large interactive surfaces, such as tabletops are well explored (e.g., $[15,19,26,30,38,40])$. However, with small-sized interactive surfaces, such as with tablets bi-manual touch input faces more constraints. For example, in addition to screen sizes being insufficient, it is easier to cause undesired screen occlusion. Yoon et al. [40] have studied how pen and touch can be used in combination to compensate insufficient space on a tablet device for making annotations by tearing the digital paper and creating additional space. An approach to overcome interaction constraints with uni-modal solutions without introducing additional user interactions is the integration of alternative input modalities.

A trend resulting from advances in sensing technologies is expanding the interaction space of touch screen devices, such as mobiles and tablets, above and around the actual touchsensitive screens (e.g., [25, 31, 39, 41]). This trend shows promise to address many of the limitations in unimodal touch interaction with interactive surfaces by supporting alternative input techniques and combining them with touch input. However, the expanded interaction space poses a challenge for gestural interaction design. A mismatch between designed gestures and how real users would want to use gestures in interactions (i.e., the gulf of execution [33]) is one of the issues designers and developers encounter when designing gestural interaction for novel and future interaction spaces. In order to reduce a potentially large gulf of execution many researchers have adopted the user-elicitation method by Wobbrock et al. [37], which is based on the guessability technique [32, 36], which was also applied to design pen input on touch screens in combination with mid-air gestures $[8,13]$.

\section{Contextual Mid-Air Input}

Earlier research in tabletop interaction design (e.g., [24, 35]) has already recognized the constraints of planar interaction spaces, and thus, the space above the tabletop and how it could be used to add depth and continuity to interactions has been studied. For example, Marquardt et al. [29] explored combining touch on tabletops and mid-air gestures above tabletop screens towards a continuous interaction space for tabletop interaction. Annett et al. [6] made use of proximity information to contextualize tabletop interaction, such as distinguishing and adapting to left and right hand usage.

More recently, mid-air and touch input has been proposed for various other devices and contexts, such as addressing typical issues with small-sized screens and occlusion. Chen et al. [17], for example, suggested to combine pre-touch or post-touch gestures with touch allowing alternative forms to perform operations, such as to zoom by tabbing the screen followed by circling with the finger above the screen. Hinckley et al. [25] suggested to adapt touch interfaces depending on the posture of the approaching hand, for example to differentiate a two-finger-zoom intention from a thumb-tab intention on the screen and thus reduce information overload on the screen. Aslan et al. [7] have explored the user experience of pre-touch proxemic interactions with touch targets, arguing that the design space should move from still graphics towards proxemic target behaviors.

Thus, previous research streams (e.g., $[9,8,7])$ have established users' interest in (i) pre-touch proxemic input and (ii) combinations of mid-air input and pen input. However, research has focussed on the conceptual design of mid-air gestures performed with the non-dominant hand while drawing on 
a tablet with a pen. Some researchers have also implemented prototypes (e.g., [8, 27]), but so far none seem to have explored bi-manual pen+mid-air input (as depicted in Figure 1) in an empirical study. In order to close this gap in research, we have developed a prototype and studied with users how they experienced pen+mid-air input, focusing on proxemic hand input aside the screen and pen input above the screen. In our own previous research (e.g., $[8,13])$ we have already studied the design space of pen+mid-air input, including literature reviews and contextual inquiries to improve our understanding of relevant operations. Thus, our decision to focus, in the research at hand, on zooming and panning as exemplary operations is based on our previous inquiries.

In the next section, we describe in detail the prototype system and the input techniques that it enables in detail.

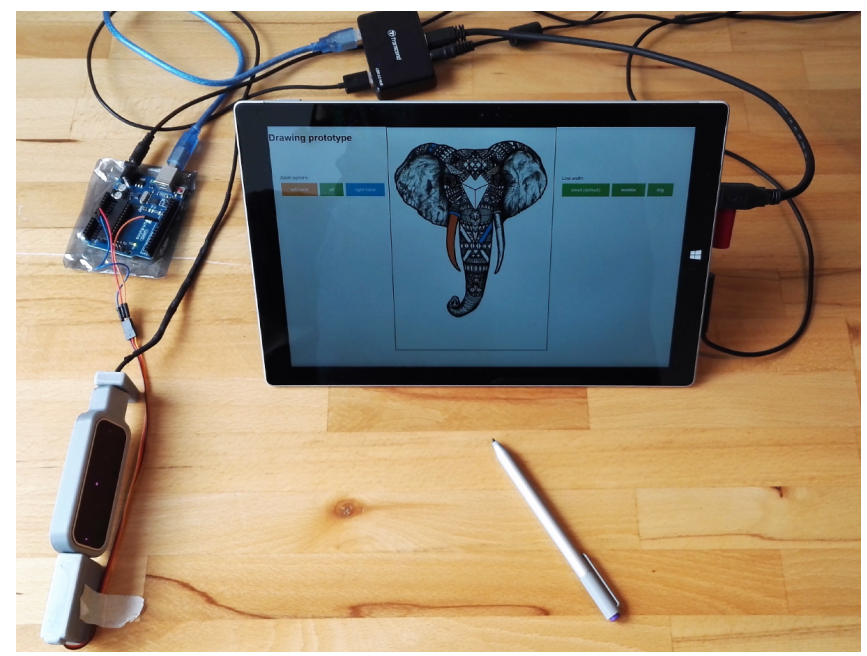

Figure 2. Overview of the prototype consisting of a drawing application which runs on a touch screen device (a Microsoft Surface device), a digital pen, a micro-controller (i.e., an Arduino board), and a "robotic arm" to actuate a Leap Motion sensor's orientation.

\section{PROTOTYPE}

Figure 2 provides an overview of all parts of the prototype, including a "screenshot" of the drawing application, which combines and utilizes all the hardware.

\section{LeapArm 2.0 Prototype}

The original LeapArm prototype [9] could actuate a Leap Motion controller's orientation using two servomotors in all directions. Inspired by the original system we decided to build a version 2.0 that is customized for asided and above screen input and thus requires only one servo motor to actuate the $3 \mathrm{D}$ controllers orientation either aside or above the touch screen (see Figure 3). The LeapArm 2.0 is smaller and more compact than the original version. The servomotor, which is used to move the Leap Motion's orientation is inside a 3D printed body consisting of 3 parts. Two ball bearings are utilized to connect all three parts and to be able to easily move the middle part in which a Leap Motion sensor is embedded. LeapArm 2.0 is connected to an Arduino Uno board. Ultimately, LeapArm is a small robotic mount for the Leap Motion controller, which we build to actuate via software the Leap Motion's orientations, and thus to dynamically expand the sensory space spanned by the Leap Motion controller. An alternative would have been to use multiple Leap Motion controllers; however, in our best of knowledge the SDK supports only one controller per PC.

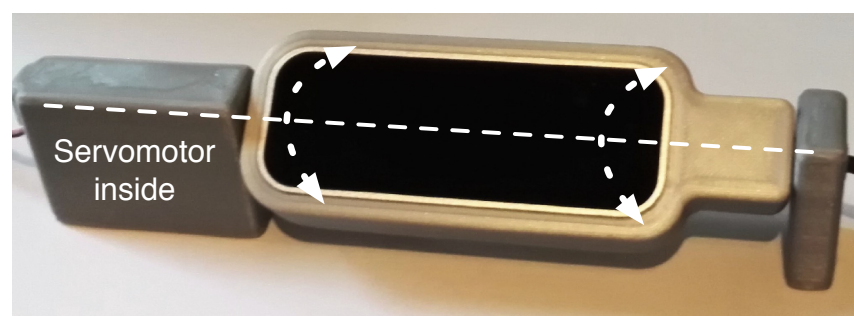

Figure 3. The LeapArm 2.0 prototype is a 3D printed "robotic arm" with an inbuilt servomotor, which can be controlled to actuate the orientation of the embedded 3D controller (i.e., a Leap motion sensor).

The Leap Motion itself [4] is an exemplary (and widely used) optical tracking system. It is customized to recognize hand and finger movement of users who are typically in a seated position in front of a screen. The controller needs to be put on the desktop beside or in front of a computer screen. The range of the Leap Motion is limited to a space from approximately 25 to 600 millimeters above the device and a field of view of about 150 degrees with recognition rates being worse in the borders of the sensory space than the center of the space [5].

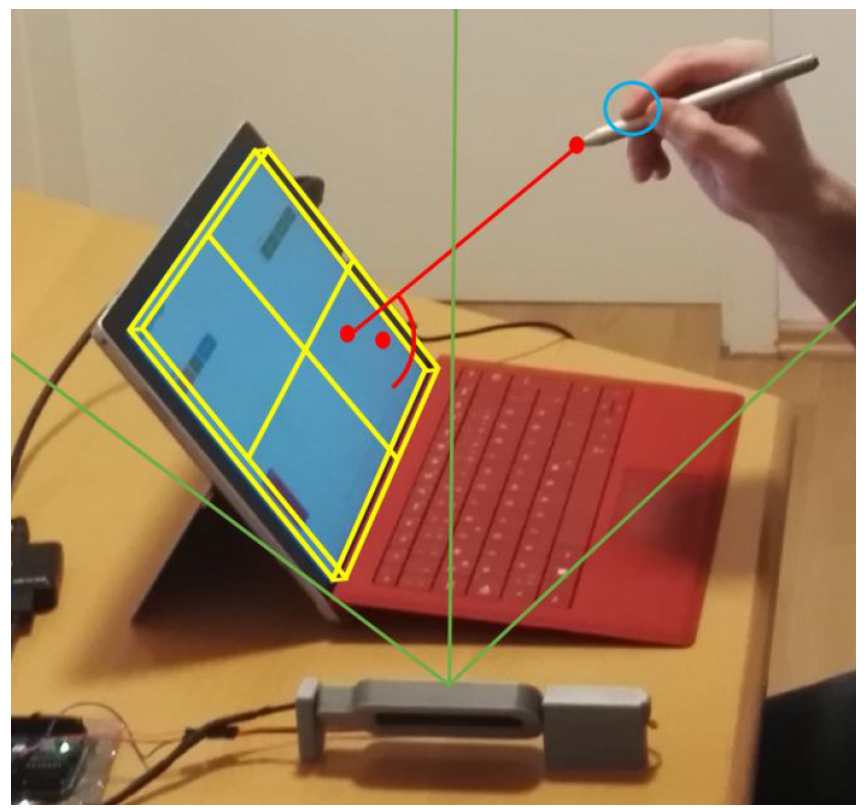

Figure 4. Leap Motion interaction space and touch-screen input space need to be synchronized to allow pre-touch proxemic 3D interaction above the screen.

\section{Drawing Application}

In order to integrate the Leap Motion sensor, the LeapArm actuator, a pen, and a touchscreen we developed a web application, which first synchronizes the Leap Motion input space with the touch screen input space. We have replicated the 
procedure proposed by Aslan and Andrè [7], which requires a short calibration task in which users are asked to touch targets on three corners of the touch screen to map 2D screen positions with 3D finger positions, allowing to calculate proximity of finger positions in 3D to touch targets on the screen (see Figure 4). Furthermore, to control the LeapArm via an Arduino board we utilized the Firmata Protocol [1], Node.js [3], and the Johny-Five [2] framework. The drawing application is based on Javascript and uses an HTML5 canvas to allow real time drawing with a digital pen.

The application implements two new input techniques to manipulate the canvas (i.e., zoom in and out, or move/scroll the canvas). Users can press a button on the application to control the LeapArm and move the Leap Motion's orientation above the screen or aside the screen.

When the orientation is above the screen users can zoom with their preferred hand that is holding the pen through changing their hand's distance to the screen (i.e., moving up and down). Users can also move/pan the canvas by moving to the left or to the right (i.e., when the hand moves above the screen to the right the canvas moves/scrolls to the left). The above screen interaction is a compound form of interaction style. When a user moves towards a target, change in distance and orientation of the user's finger in relation to the target is used to zoom and move the canvas. The goal is to have the user transition between inking in a zoomed in mode and perceiving the overall drawing and finding the next spot to ink in a zoomed out mode. We have realized this by stopping any zoom or pan action once the distance to the screen/drawing is less than 2 inches (which we defined base on our own experience/exploration). Consequently while drawing the image does not move.

When the orientation is aside the screen users can zoom with their hand that is not holding the pen through changing their hand's distance to the screen (i.e., moving left and right). Figure 1 depicts both techniques. We also implemented a button on the GUI, which can be pressed to freeze the drawing in cases users want the canvas to freeze for a while independent from their distance to the screen.

\section{USER STUDY}

In order to explore the UX of drawing and using the new input techniques, which allow input above and aside the screen, we conducted a repeated measurement study comparing the new techniques against a baseline.

\section{Participants, Apparatus, and Procedure}

Twenty right-handed participants (10f, 10m) between 21 and 54 years of age (mean age of 26.4) were recruited. We chose to only use right-handed participants to reduce any handedness based bias, including a bias possibly caused by changing/switching the configuration of the setup. Fourteen participants stated to have prior experience using a digital pen on a touch screen and six reported to have no experience in drawing on a touch screen with a digital pen. All participants were asked to complete the same drawing/inking tasks in counter-balanced order (to address learning bias due to order and repetition) with both interaction modalities (i.e., baseline and new modality).
After each task participants were asked to fill out the attrakDiff questionnaire [23] providing explicit input on how the interaction technique was experienced. At the end of both tasks a semi-structured interview was conducted in order to get further insides, including reasons for why participants rated one modality over the other.

Before participants were asked to complete the task with the new modality, they watched a short video tutorial demonstrating the above and aside input technique. We did this to make sure that all participants received exactly the same instructions/help.

\section{Inking Task}

Each participant had to ink parts of the same elephant drawing (see Figure 2). We chose to use the elephant (Polynesian tattoo) motif since it consisted of delicate structures and would required zooming in and out. The parts that needed to be filled with color were marked either orange or blue. We told participants to use the orange zoom mode (i.e., left hand zoom mode) for filling in the orange parts and the blue zoom mode (i.e., right hand zoom mode) for filling in the blue parts.

Participants could switch between the orange and blue zoom mode whenever they wanted. We used the color codes to have participants try out both zooming techniques (i.e., above screen zoom and aside screen zoom) that are enabled by our prototype. Participants could take as much time as they needed to fill in all marked sections in the drawing. In the baseline condition we asked participants to ink the the same image (i.e., both orange and blue sections) using the standard Microsoft Paint application. Participant could zoom via multi-touch or the zoom slider and button provided by Paint.

\section{UX Questionnaire and Observations}

The attrakDiff questionnaire measures pragmatic quality (PQ), which is a measure for perceived traditional usability and hedonic quality (HQ), which results from a combination of HQS, HQI, and ATT. HQS measures the perceived ability of a product to meet a person's desire for self-improvement, HQI measures the perceived ability of a product to communicate a valuable identity to others, and ATT measures overall attractiveness. We chose the attrakDiff questionnaire because it is widely used in research and industry, providing an overview of a product's or technique's perceived qualities beyond traditional usability.

In addition to observing participants during each session, sessions were video recorded for a post-hoc analysis. The semistructured interview, which was conducted at the end with each participant included questions, such as "Which interaction technique did you perceive as most comfortable and why?". We also differentiated between aside and above input for zooming, in order to understand which technique participants preferred for zooming and why.

Since we occasionally experienced tracking issues with the Leap Motion sensor, we decided to also ask a question, considering if participants encountered any technical issues with the prototype and if these issues influenced their ratings and experience. 


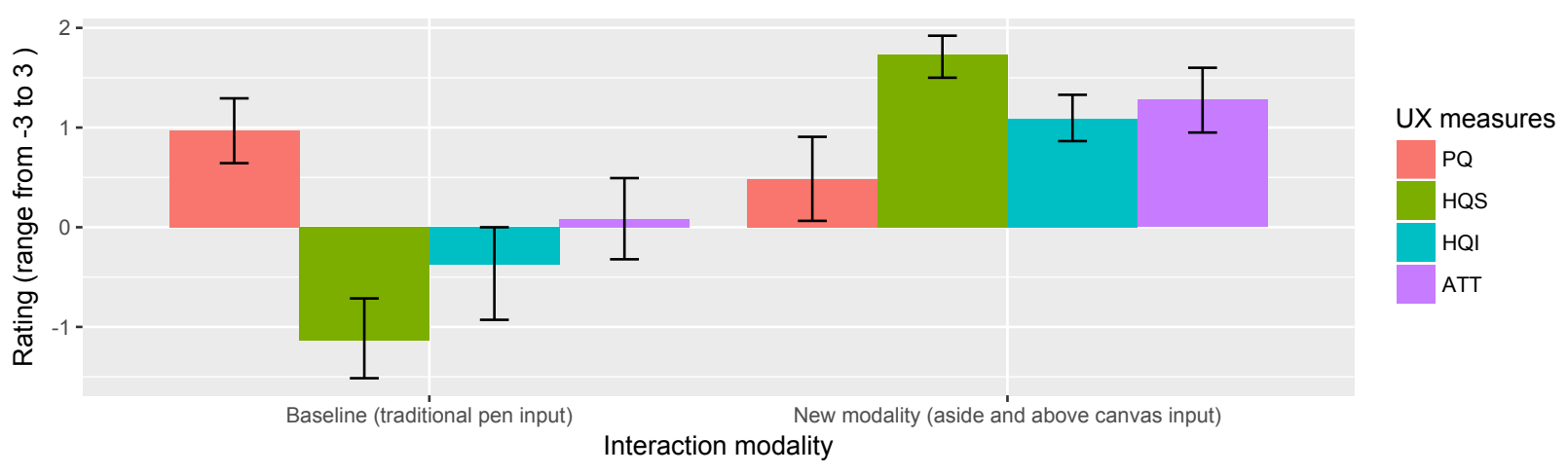

Figure 5. Mean ratings for the measured UX constructs PQ, HQS, HQI, and ATT explaining pragmatic (i.e., perceived traditional usablity) and hedonic qualities. Error bars denote $95 \%$ confidence intervals.

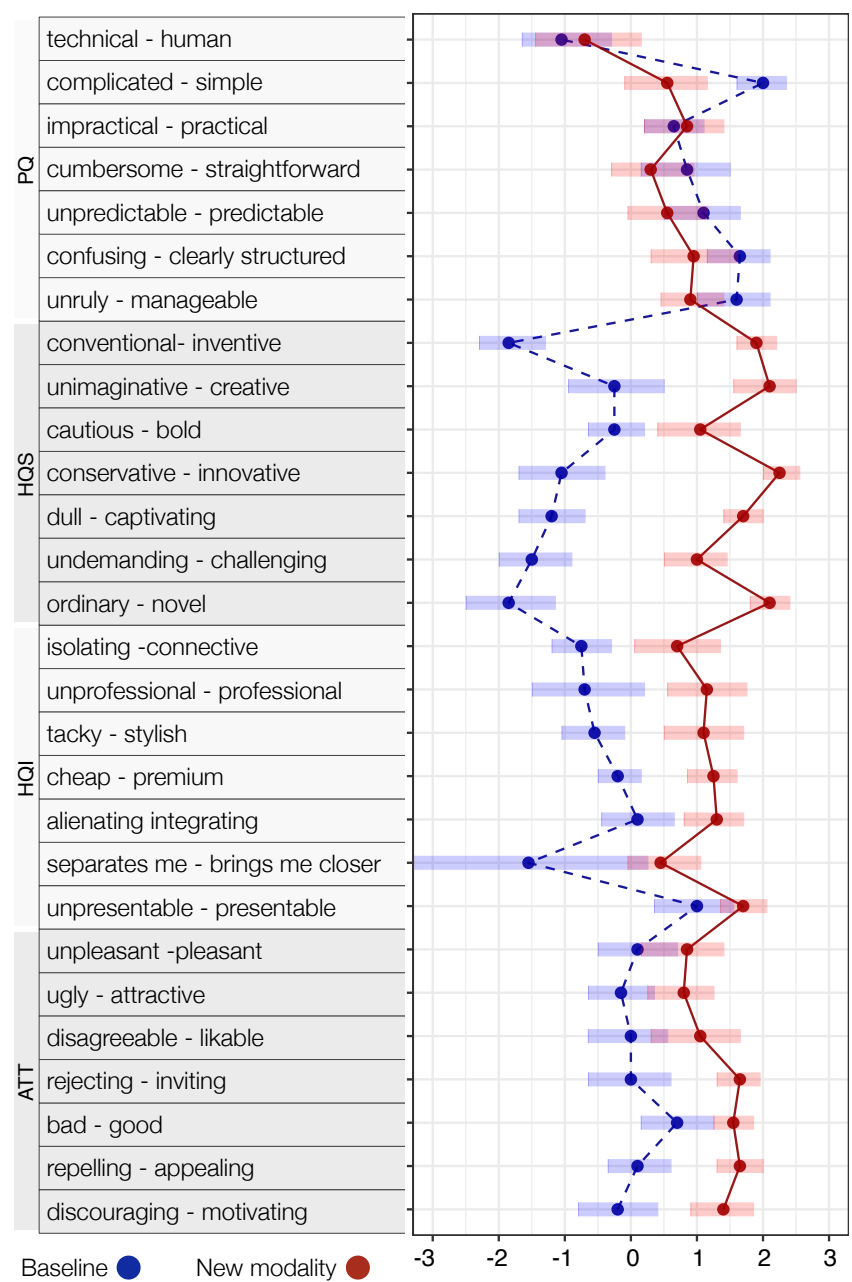

Error bars/rectangles denote 95\% confidence intervals

Figure 6. Overview of UX self-reports for all items of the attrakDiff questionnaire.

\section{Results}

In the following, we start by presenting general trends based on graphical presentations of the data considering UX measurements. Then, results will be interpreted based on fitting a statistical model to the data (i.e., results of statistical test will be provided). Afterwards, we describe the results of the post-hoc interviews providing insights on reasons of specific results and observations.

\section{General Trends}

Figure 5 presents the mean values for all four measured UX constructs (i.e., PQ, HQS, HQI and ATT). It seems that there are differences in how the new modality was experienced compared to the baseline mainly in constructs explaining hedonic qualities. The highest difference seems in HQS (i.e., the hedonic quality that is associated with the perceived ability of a product/technique to meet a person's desire for selfimprovement) and the lowest difference seems to exist in PQ (i.e., perceived traditional usability).

Figure 6 depicts for each construct mean values for all constituting seven items, detailing how participants experienced the drawing task for both interaction modalities. Considering HQS, the new modality was experienced, as expected, as novel and innovative, but also as creative and captivating. It seems that the new modality received higher ratings for all items explaining the modalities' hedonic quality, including items not related with the modality's novelty aspect, such as being perceived as more professional, inviting, appealing, connective, and thus potentially communicating a valuable identity to others (HQI). While the differences in ratings for the seven items constituting to ATT seem less than in those constituting to HQS, and slightly less than in those constituting to HQI, the overall difference seems systematic with the new modality having been perceived as consistently more attractive.

In contrast, the baseline modality seems to have received slightly higher ratings considering items explaining the modalities pragmatic quality (i.e., perceived traditional usability), such as simplicity.

Last but not least, Figure 7 shows the difference in how the new modality is perceived different from the baseline consid- 
ering pragmatic quality (PQ) and hedonic quality (HQ) (i.e., combination/aggregation of HQS, HQI, and ATT).

Before we present the qualitative analysis, which aims to provide additional insights on why participants seem to have considered the new modality superior considering hedonic qualities we check the significance of differences for all four constructs by computing statistical tests. Based on the graphical presentation of the data we would expect significant difference in hedonic qualities since nearly all items seem to have received higher ratings in mean, but it seems unclear if there is a significant difference in pragmatic quality (PQ).

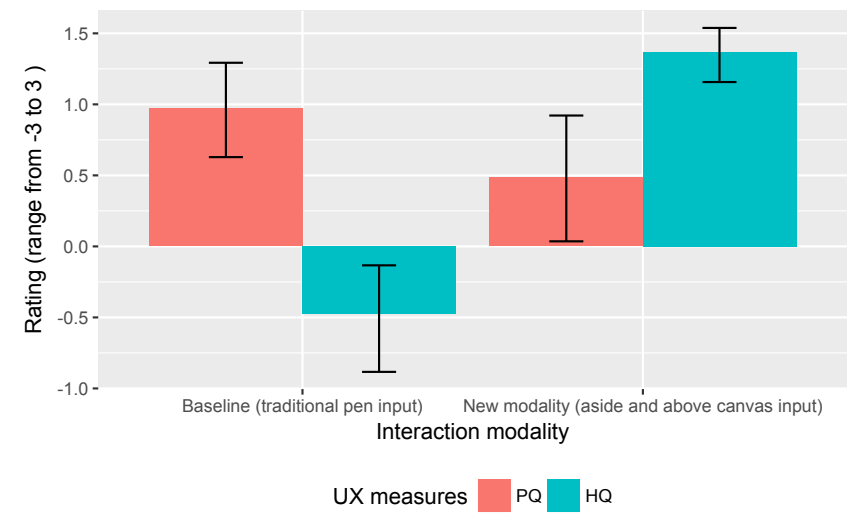

Figure 7. Difference between the the new modality and the baseline considering PQ and HQ. Error bars denote $95 \%$ confidence intervals.

\section{Statistical Analysis}

In order to compare participants ratings for PQ, HQS, HQI, and ATT for both modalities we conducted paired-samples $\mathrm{t}$-tests. While the difference in PQ was non-significant $(\mathrm{t}=-$ $1.67, \mathrm{p}=.11, \mathrm{r}=.36)$ the differences in HQS $(\mathrm{t}=11.89, \mathrm{p}<.001$, $\mathrm{r}=.94)$ in HQI $(\mathrm{t}=4.52, \mathrm{p}<.001, \mathrm{r}=.72)$, and in ATT $(\mathrm{t}=4.12$, $\mathrm{p}<.001, \mathrm{r}=.68$ ) were significant. The difference in HQ was also significant $(\mathrm{t}=7.93, \mathrm{p}<.001, \mathrm{r}=.88)$.

Consequently, participants seem to have perceived a significant difference considering hedonic qualities of the new modality. In contrast the study results do not show a significant difference considering pragmatic quality (i.e., perceived traditional usability). Overall, participants considered the new modality significantly more attractive and desirable than the baseline modality.

\section{Qualitative Analysis}

At the end we had asked participants which technique they preferred most and 11 participants preferred the aside input, 5 the above input, and 4 preferred the baseline condition.

In order to deepen our understanding of why participants attributed higher hedonic qualities to the new modality we analyzed the qualitative data collected through semi-structure interviews, video recording, and observations applying a thematic analysis [16]. The analysis focused on how people described their experience considering (i) the baseline condition, (ii) aside the screen input for zooming, and (iii) above the screen input for a combination of zooming and and panning/scrolling.

First, we transcribed relevant comments (i.e., direct references to the interaction techniques) in the video recordings and added these comments to the data collected through the post-hoc interviews. Then words and sentences that occurred repeatedly or were explicitly mentioned as important by participants were marked. Afterwards three mind-maps were generated. Lastly, the mind maps were diverged into common descriptors (e.g., by merging similar branches in the mindmap) to reduce redundant information in the mind-maps and to only identify main themes. Figure 8 depicts the resulting themes for each interaction technique.

\section{Baseline Condition}

The themes that came up when participants spoke about the baseline condition were stability, routine and effort. Participants who preferred the baseline condition argued that based on their previous experience they already have a drawing routine, which they would not have to change. Others mentioned stability since in the baseline condition zooming is perceived as more stable while in the other condition "any" mid-air hand movement may cause the canvas to zoom. On the other hand, participants argued that the baseline condition requires more effort since the task has to be completed in a sequential fashion and with participants having to change their locus too often compared to the other interaction modalities.

\section{Above Input}

The themes that emerged about the above input condition were preferred hand, training, and supporting. Participants stated that the interaction modality was challenging but not too challenging, and that it felt like self-training, which was perceived as supporting in the sense that it seemed to improve/increase their competence. Participants argued that there is a lot of potential in the above input modality. Others spoke about how it was a simple interaction modality, since it only required control over the preferred hand.

\section{Aside Input}

Stimulating, dynamic use and useful work process were the themes associated with the aside input modality. Participants perceived the aside modality as motivating, and overall as stimulating. We believe that participants associated stimulation with the aside modality since the modality required bi-manual engagement and overall more bodily involvement. The work process is split into both hands and therefore considered useful. Furthermore, the interaction itself was described as dynamic but also very direct.

In summary, the thematic analysis provided insight into what qualities participants associated with each modality and why they preferred one modality over an other. Overall, the baseline condition was associated with positive attributes because participants considered it already as a routine that they have adopted. But many participants (15 of 20) still have preferred the aside or above input modality arguing that it either seemed to provide potential to improve their own competence or that it was considered as stimulating and enabling a dynamic and useful work process. 


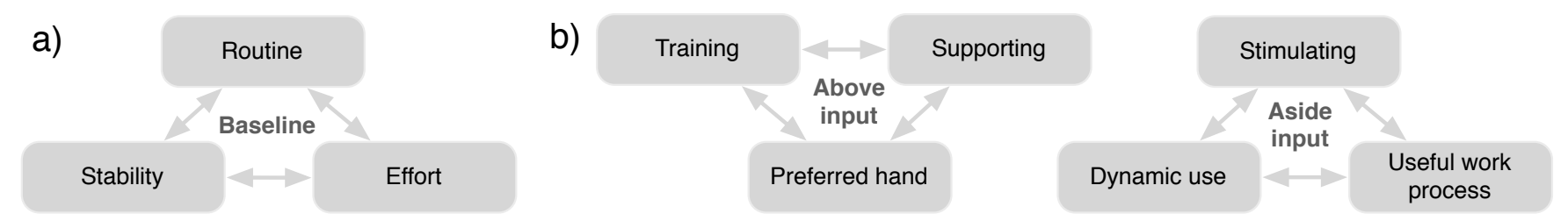

Figure 8. Result of thematic analysis for a) the baseline condition and b) the new modality separated by the concrete input techniques (i.e., mid-air pen input above screen and hand input aside screen input).

\section{DISCUSSION}

We have argued that digitalization is changing many traditional practices and that drawing is one of these practices, which is being transformed and hopefully enriched by new digital and malleable materials, such as digital canvases and digital ink. Often, a first step in introducing new materials is in replicated known interaction metaphors and applying skeuomorphism (e.g., simulating behavior and perception of known materials and concepts) in order to make it easy for users to adopt a new technique or technology. Thus, it is not surprising that pen-based interaction on touch screens resembles pen based input on paper.

However, to "fully" make use of the new materials potential new interaction techniques may be required. In this paper, we presented a prototype which enables mid-air proxemic input above and aside a touch screen (and digital canvas). Moreover, we have explored how users perceive interacting with their non-preferred hand aside the screen to zoom in and out a drawing, and with their pen holding preferred hand above the screen performing compound action (i.e., zooming an panning a drawing) before and after inking. We believe that the new larger interaction space, which includes mid-air sensory space aside and above a touch screen is open for many more techniques than what we have explored in this paper. We think that new techniques enable and stimulate human thought, and thus, we will study more techniques including, for example, manipulations of digital ink in our future work.

Nevertheless, we believe to have demonstrated with the work at hand, based on exemplary techniques and an exemplary inking task, the space's potential to change users experiences to the better. Ultimately, we believe and hope that the combination of the UX questionnaire and the thematic analysis has provided valuable insights, not only for us but also for fellow researchers, inspiring further studies of new aside and above screen input techniques and applications.

Despite the positive resonance from users considering the prototype, the research we presented in this paper faces some limitations related to technical issues, which we encountered with tracking finger positions with the Leap Motion sensor. We believe the main reason was in how we positioned and oriented the Leap Motion, which was different from how it is supposed to be used (i.e., facing upwards without any physical obstacles in the field of view of the controller). We placed the Leap Motion aside the screen with an orientation either towards the screen or towards the non-preferred hand, which was in resting on the table aside the screen. The screen itself may have reflected some light and overall we encountered oc- casional tracking issues, which we couldn't predict nor control. However, what we have done to get insights is to ask participants at the end of the study if they encountered technical issues and if these issues influenced their experience. Only three of the twenty participants stated that their experience and ratings were influenced by technical issues that they encountered, stating that they either perceived the tracking as not seamless or its latency as too high. One participant argued that if she had only viewed the instructional video, her ratings might have been more positive but the technical issues that she encountered influenced her experience.

Consequently, we assume that with a more robust finger tracking the new interaction modality might have received slightly better user ratings. Thus, in our future work we will consider other tracking technologies, including interactive gloves. In our experience drawing is not only a professional activity but also an activity performed at leisure time. Furthermore, some fellow researcher have already shown how touch and midair input can be combined to create short cuts and improve interaction performance (e.g., [10, 17, 26]). Thus, we have exclusively focused on exploring perceived user experience and did not study traditional performance measures, such as task completion times or interaction accuracy.

We believe, a potential next step could still be to identify performance relevant tasks and compare performance of different aside and above input techniques to baseline conditions. Indeed one participant explicitly stated that they see a potential in the new techniques for productivity/office related applications and to improve performance. Although, we studied above and aside input individually when analyzing user comments the fact that we did not compare aside input and above input with each other utilizing the UX questionnaire may be considered an additional limitation of the study.

A concrete next future step that we plan in order to further improve the interaction space for drawing related tasks with a pen on touch screens is to augment the digital pen with additional actuators, such as light emitting diodes and a vibrotactile motor. Doing this would allow to deal with the increasing interaction complexity of the expanded interaction space by improving mappings. For example, the pen itself could display the current ink color or provide contextual information to interactive elements on the screen or associated with gestures performed with the non-preferred hand before touch events have to be performed and while the pen is above the screen. 


\section{CONCLUSION}

First, we presented a prototype consisting of a touch-device (i.e., Microsoft Surface), a 3D controller (i.e., Leap Motion), a "robotic arm" to actuate the orientation of the 3D controller above or aside the screen, which enables pen and hand input mid-air above and aside a touch screen. Then, we reported on an empirical study with twenty participants comparing the UX of using two interaction modalities for an inking task. One modality was the baseline, in which participants could use only hand and pen based touch input, and the other condition included aside and above screen input techniques for zooming and panning the drawing. We found significant differences in UX, showing that the new modality's UX was superior considering hedonic qualities, such as overall attractiveness of the interaction techniques. Overall participants considered the new interaction techniques more desirable and saw a higher potential in the new techniques to, for example help them improve themselves. Furthermore, a thematic analysis identified details in differences and qualities associated with above and aside input, showing for example that (i) aside input was considered as a stimulating and useful work process since it engaged both hands and (ii) above input was considered supporting the interaction by combining/merging necessary actions (i.e., zoom and pan at the same time).

\section{ACKNOWLEDGMENTS}

We thank Julian Kraus for his contributions in adapting the LeapArm system.

\section{REFERENCES}

1. 2018. GitHub. firmata/arduino: Firmata firmware for arduino. https://github.com/firmata/arduino.. (2018). Accessed: 2018-01-15.

2. 2018. Johnny-Five: The javascript robotics \& iot platform. http://johnny-five.io/. (2018). Accessed: 2018-08-21.

3. 2018. Node.js Foundation. https://nodejs.org/. (2018). Accessed: 2018-01-15.

4. 2018. Reach into virtual reality with your bare hands. https://www . leapmotion. com/. (2018). Accessed: 2018-01-15.

5. Vamsi Kiran Adhikarla, Jaka Sodnik, Peter Szolgay, and Grega Jakus. 2015. Exploring Direct 3D Interaction for Full Horizontal Parallax Light Field Displays Using Leap Motion Controller. Sensors 15, 4 (2015), 8642-8663. DOI : http://dx.doi . org/10.3390/s150408642

6. Michelle Annett, Tovi Grossman, Daniel Wigdor, and George Fitzmaurice. 2011. Medusa: A Proximity-aware Multi-touch Tabletop. In Proc. of (UIST '11). ACM, 337-346.

7. Ilhan Aslan and Elisabeth André. 2017. Pre-touch Proxemics: Moving the Design Space of Touch Targets from Still Graphics Towards Proxemic Behaviors. In Proceedings of the 19th ACM International Conference on Multimodal Interaction (ICMI 2017). ACM, New York, NY, USA, 101-109. DOI :

http://dx.doi.org/10.1145/3136755.3136808
8. Ilhan Aslan, Ida Buchwald, Philipp Koytek, and Elisabeth André. 2016a. Pen + Mid-Air: An Exploration of Mid-Air Gestures to Complement Pen Input on Tablets. In Proceedings of the 9th Nordic Conference on Human-Computer Interaction (NordiCHI '16). ACM, New York, NY, USA, Article 1, 10 pages. DOI : http://dx.doi.org/10.1145/2971485.2971511

9. Ilhan Aslan, Julian Kraus, and Elisabeth André. 2016b. LeapArm-Facilitating Sensory Spaces for Mid-air Gestural Interaction. In Proceedings of the 9th Nordic Conference on Human-Computer Interaction (NordiCHI '16). ACM, New York, NY, USA, Article 106, 6 pages. DOI : http://dx.doi .org/10.1145/2971485.2996741

10. Ilhan Aslan, Alina Krischkowsky, Alexander Meschtscherjakov, Martin Wuchse, and Manfred Tscheligi. 2015a. A Leap for Touch: Proximity Sensitive Touch Targets in Cars. In Proceedings of the 7th International Conference on Automotive User Interfaces and Interactive Vehicular Applications (AutomotiveUI '15). ACM, New York, NY, USA, 39-46. DOI : http://dx.doi.org/10.1145/2799250.2799273

11. Ilhan Aslan, Thomas Meneweger, Verena Fuchsberger, and Manfred Tscheligi. 2015b. Sharing Touch Interfaces: Proximity-Sensitive Touch Targets for Tablet-Mediated Collaboration. In Proceedings of the 2015 ACM on International Conference on Multimodal Interaction (ICMI '15). ACM, New York, NY, USA, 279-286. DOI : http://dx.doi.org/10.1145/2818346.2820740

12. Ilhan Aslan, Tabea Schmidt, Jens Woehrle, Lukas Vogel, and Elisabeth André. 2018a. Pen + Mid-Air Gestures: Eliciting Contextual Gestures. In Proceedings of the 20th ACM International Conference on Multimodal Interaction (ICMI'18). ACM, New York, NY, USA, 135-144. DOI : http://dx.doi.org/10.1145/3242969.3242979

13. Ilhan Aslan, Tabea Schmidt, Jens Woehrle, Lukas Vogel, and Elisabeth André. 2018b. Pen + Mid-Air Gestures: Eliciting Contextual Gestures. In Proceedings of the 2018 on International Conference on Multimodal Interaction (ICMI '18). ACM, New York, NY, USA, 135-144. DOI : http://dx.doi.org/10.1145/3242969. 3242979

14. Ilhan Aslan, Andreas Uhl, Alexander Meschtscherjakov, and Manfred Tscheligi. 2016. Design and Exploration of Mid-Air Authentication Gestures. ACM Trans. Interact. Intell. Syst. 6, 3, Article 23 (Sept. 2016), 22 pages. DOI: http://dx.doi.org/10.1145/2832919

15. Peter Brandl, Clifton Forlines, Daniel Wigdor, Michael Haller, and Chia Shen. 2008. Combining and Measuring the Benefits of Bimanual Pen and Direct-touch Interaction on Horizontal Interfaces. In Proceedings of the Working Conference on Advanced Visual Interfaces (AVI '08). ACM, New York, NY, USA, 154-161. DOI : http://dx.doi.org/10.1145/1385569.1385595

16. Virginia Braun and Victoria Clarke. 2006. Using thematic analysis in psychology. Qualitative Research in Psychology 3, 2 (2006), 77-101. DOI : http://dx.doi.org/10.1191/1478088706qp063oa 
17. Xiang 'Anthony' Chen, Julia Schwarz, Chris Harrison, Jennifer Mankoff, and Scott E. Hudson. 2014. Air+Touch: Interweaving Touch \&\#38; In-air Gestures. In Proceedings of the 27th Annual ACM Symposium on User Interface Software and Technology (UIST '14). ACM, New York, NY, USA, 519-525. DOI : http://dx. doi.org/10.1145/2642918.2647392

18. Dustin Freeman, Ramadevi Vennelakanti, and Sriganesh Madhvanath. 2012. Freehand pose-based Gestural Interaction: Studies and implications for interface design. In Proc. of IHCI'12. IEEE, 1-6.

19. Mathias Frisch, Ricardo Langner, and Raimund Dachselt. 2011. Neat: A Set of Flexible Tools and Gestures for Layout Tasks on Interactive Displays. In Proceedings of the ACM International Conference on Interactive Tabletops and Surfaces (ITS '11). ACM, New York, NY, USA, $1-10$. DOI :

http://dx.doi.org/10.1145/2076354.2076356

20. Darren Guinness, Alvin Jude, G. Michael Poor, and Ashley Dover. 2015. Models for Rested Touchless Gestural Interaction. In Proceedings of the $3 r d$ ACM Symposium on Spatial User Interaction (SUI '15). ACM, New York, NY, USA, 34-43. DOI :

http://dx.doi.org/10.1145/2788940.2788948

21. Jože Guna, Grega Jakus, Matevž Pogačnik, Sašo Tomažič, and Jaka Sodnik. 2014. An analysis of the precision and reliability of the leap motion sensor and its suitability for static and dynamic tracking. Sensors 14, 2 (2014), 3702-3720.

22. Sean Gustafson, Daniel Bierwirth, and Patrick Baudisch. 2010. Imaginary Interfaces: Spatial Interaction with Empty Hands and Without Visual Feedback. In Proceedings of the 23Nd Annual ACM Symposium on User Interface Software and Technology (UIST'10). ACM, New York, NY, USA, 3-12. DOI: http://dx.doi.org/10.1145/1866029.1866033

23. Marc Hassenzahl, Michael Burmester, and Franz Koller. 2003. AttrakDiff: Ein Fragebogen zur Messung wahrgenommener hedonischer und pragmatischer Qualität. In Mensch \& Computer 2003. Springer.

24. Otmar Hilliges, Shahram Izadi, Andrew D. Wilson, Steve Hodges, Armando Garcia-Mendoza, and Andreas Butz. 2009. Interactions in the Air: Adding Further Depth to Interactive Tabletops. In Proceedings of the 22Nd Annual ACM Symposium on User Interface Software and Technology (UIST '09). ACM, New York, NY, USA, 139-148. DOI :

http://dx.doi.org/10.1145/1622176.1622203

25. Ken Hinckley, Seongkook Heo, Michel Pahud, Christian Holz, Hrvoje Benko, Abigail Sellen, Richard Banks, Kenton O'Hara, Gavin Smyth, and William Buxton. 2016. Pre-Touch Sensing for Mobile Interaction. In Proceedings of the $2016 \mathrm{CHI}$ Conference on Human Factors in Computing Systems (CHI '16). ACM, New York, NY, USA, 2869-2881. DOI : http://dx.doi.org/10.1145/2858036.2858095
26. Ken Hinckley, Koji Yatani, Michel Pahud, Nicole Coddington, Jenny Rodenhouse, Andy Wilson, Hrvoje Benko, and Bill Buxton. Pen + Touch $=$ New Tools. In Proc. of UIST '10. ACM, 10.

27. Sungjae Hwang, Andrea Bianchi, Myungwook Ahn, and Kwangyun Wohn. 2013. MagPen: Magnetically Driven Pen Interactions on and Around Conventional Smartphones. In Proceedings of the 15th International Conference on Human-computer Interaction with Mobile Devices and Services (MobileHCI '13). ACM, New York, NY, USA, 412-415. DOI :

http://dx.doi.org/10.1145/2493190.2493194

28. Sven Kratz and Michael Rohs. 2009. HoverFlow: Expanding the Design Space of Around-device Interaction. In Proc. of (MobileHCI '09). ACM, New York, NY, USA, Article 4, 8 pages. DOI : http://dx.doi.org/10.1145/1613858.1613864

29. Nicolai Marquardt, Ricardo Jota, Saul Greenberg, and Joaquim A Jorge. 2011. The continuous interaction space: interaction techniques unifying touch and gesture on and above a digital surface. In INTERACT 2011. Springer.

30. Fabrice Matulic and Moira C. Norrie. 2013. Pen and Touch Gestural Environment for Document Editing on Interactive Tabletops. In Proceedings of the 2013 ACM International Conference on Interactive Tabletops and Surfaces (ITS '13). ACM, New York, NY, USA, 41-50. DOI: http://dx.doi.org/10.1145/2512349.2512802

31. Rajalakshmi Nandakumar, Vikram Iyer, Desney Tan, and Shyamnath Gollakota. 2016. FingerIO: Using Active Sonar for Fine-Grained Finger Tracking. In Proceedings of the 2016 CHI Conference on Human Factors in Computing Systems (CHI '16). ACM, New York, NY, USA, 1515-1525. DOI: http://dx.doi.org/10.1145/2858036.2858580

32. Michael Nielsen, Moritz Störring, Thomas B Moeslund, and Erik Granum. 2003. A procedure for developing intuitive and ergonomic gesture interfaces for HCI. In International Gesture Workshop. Springer, 409-420.

33. Donald A Norman and Stephen W Draper. 1986. User centered system design. Hillsdale, $N J$ (1986), 1-2.

34. Juan Pablo Wachs, Mathias Kölsch, Helman Stern, and Yael Edan. 2011. Vision-based Hand-gesture Applications. Commun. ACM 54, 2 (Feb. 2011), 60-71.

35. Andrew D. Wilson, Shahram Izadi, Otmar Hilliges, Armando Garcia-Mendoza, and David Kirk. 2008. Bringing Physics to the Surface. In Proceedings of the 21st Annual ACM Symposium on User Interface Software and Technology (UIST '08). ACM, New York, NY, USA, 67-76. DOI : http://dx.doi.org/10.1145/1449715.1449728

36. Jacob O. Wobbrock, Htet Htet Aung, Brandon Rothrock, and Brad A. Myers. 2005. Maximizing the Guessability of Symbolic Input. In CHI '05 Extended Abstracts on Human Factors in Computing Systems (CHI EA '05). ACM, New York, NY, USA, 1869-1872. DOI : http://dx.doi.org/10.1145/1056808.1057043 
37. Jacob O Wobbrock, Meredith Ringel Morris, and Andrew D Wilson. 2009. User-defined gestures for surface computing. In Proceedings of the SIGCHI Conference on Human Factors in Computing Systems. ACM, 1083-1092.

38. Mike Wu, Chia Shen, Kathy Ryall, Clifton Forlines, and Ravin Balakrishnan. 2006. Gesture registration, relaxation, and reuse for multi-point direct-touch surfaces. In Horizontal Interactive Human-Computer Systems, 2006. Table Top 2006. IEEE, 8-pp.

39. Xing-Dong Yang, Tovi Grossman, Pourang Irani, and George Fitzmaurice. 2011. TouchCuts and TouchZoom: Enhanced Target Selection for Touch Displays Using Finger Proximity Sensing. In Proceedings of the SIGCHI Conference on Human Factors in Computing Systems (CHI '11). ACM, New York, NY, USA, 2585-2594. DOI : http://dx.doi.org/10.1145/1978942 . 1979319
40. Dongwook Yoon, Nicholas Chen, and François Guimbretière. 2013. TextTearing: Opening White Space for Digital Ink Annotation. In Proceedings of the 26th Annual ACM Symposium on User Interface Software and Technology (UIST'13). ACM, New York, NY, USA, 107-112. DOI :

http://dx.doi.org/10.1145/2501988.2502036

41. Chen Zhao, Ke-Yu Chen, Md Tanvir Islam Aumi, Shwetak Patel, and Matthew S. Reynolds. 2014. SideSwipe: Detecting In-air Gestures Around Mobile Devices Using Actual GSM Signal. In Proceedings of the 27th Annual ACM Symposium on User Interface Software and Technology (UIST '14). ACM, New York, NY, USA, 527-534. DOI :

http://dx.doi.org/10.1145/2642918.2647380 